\title{
Editorial
}

$\mathbf{T}$ HE PAST two decades have witnessed revolutionary advances in biomedical imaging modalities capable of providing biological and physiological information from the cellular scale to the organ level. Recent advances have also been focused on cost-effective, noninvasive, portable, and molecularimaging technologies for imaging at microscopic, mesoscopic, and macroscopic levels. These technologies have significant potential to advance biomedical research and clinical practice. They can also provide a better understanding and monitoring of physiological and functional disorders, which could lead to mainstream diagnostic technologies of the future.

\section{SCOPE OF THE SPECIAL ISSUE}

This Special Issue addresses the emerging technologies in multiparameter biomedical optical imaging and image analysis. For this exciting area, this Special Issue invited contributions in the general areas of multiparameter, multidimensional optical microscopy, confocal microscopy, optical endoscopy and spectroscopy, fluorescence imaging and tomography, diffuse reflectance and transillumination imaging, optical coherence tomography, multiphoton imaging and microscopy, photoacoustic imaging and tomography, biophotonic imaging and tomography, and image analysis (segmentation, registration, classification, etc.). As a result of this call, we received a record number of 50 submissions that were reviewed by leading experts in their respective areas. Among the many meritorious submissions, 18 were selected to highlight and represent the breakthrough research at the leading edge of rapidly emerging technologies and scientific research work with high impact and novelty.

It should also be noted that the guest editors also organized a special session "Multi-parameter Biomedical Optical Imaging" as part of 2010 IEEE International Symposium on Biomedical Imaging: From Nano to Macro, 17 April 2010, Rotterdam, The Netherlands, where several exciting papers on this topic were presented.

\section{SPECIAL ISSUE PAPERS}

The papers in this Special Issue demonstrate some of the most exciting developments in optical imaging and image analysis from tissue to cellular and molecular levels.

Eleven papers dealing with multiparameter optical imaging and image reconstruction methods for most promising modalities such as optical coherence tomography (OCT), molecular fluorescence tomography, multispectral transillumination imaging, bioluminance tomography, and photoacoustic imaging are presented first in this Special Issue. These papers demonstrate novel optical microscopic and functional imaging methods

Digital Object Identifier 10.1109/TBME.2010.2072750 for important tissue characterization and potential diagnostic applications.

Yao et al. present a simple method to numerically correct optical-axis calculation in polarization-sensitive OCT. Through experimental studies in tendon tissue, they demonstrate the application of their method to mapping the 2-D optical axis distributions in enface polarization-sensitive OCT images.

Yalavarthy et al. show that if a priori knowledge of the refractive index for the segmented regions of tissue, or even an approximated value, is used instead of assuming identical refractive index for all regions of tissue, then much accurate estimates of optical properties can be obtained.

Tian et al. address the problem of over smoothing due to Tikhonov regularization in fluorescence molecular tomography. They utilize a third-order simplified spherical harmonics approximation to radiative transfer equation to model the photon propagation within biological tissue.

D'Alessandro et al. present novel methods of recovering depth-dependent measurements from transillumination images obtained through the Nevoscope, estimating the depthdependent point spread function, and ratiometric analysis for the quantification of oxy- and deoxy-hemoglobin. The presented methods can be used for reliable quantitative analysis of multispectral Nevoscope images for early detection of angiogenesis leading to early diagnosis of skin cancers.

Liu et al. address two issues to improve the quantitative assessment of blood-oxygen saturation level: implementation of a spectral normalization technique to eliminate the spectral modulation induced by the wavelength-distance dependent point spread function of OCTs and the reduction of the spectral speckle noise due to the highly scattering blood. They assess the effectiveness of their methods using common-path OCT system.

Wang et al. introduce a dual-modality imaging system by integrating optical-resolution photoacoustic microscopy and fluorescence confocal microscopy to provide optical absorption and fluorescence contrasts simultaneously. They demonstrate simultaneous photoacoustic angiography and fluorescence lymphangiography.

Tian et al. describe the analysis of quantitative reconstruction deviation from peak wavelength shift of luminescent source and the deviation of heterogeneous mouse model for bioluminescence tomography. They discuss that the reconstruction results are significantly affected by the peak wavelength shift and deviation of anatomical structure animal models, and the model deviations exhibit much more influence than the wavelength shift on the reconstruction results.

Yu et al. reformulate bioluminescence tomography as a least absolute shrinkage and selection operator (LASSO) problem with regularization, and propose a fast reconstruction algorithm named as stage-wise fast LASSO (SwF-LASSO) is to solve this problem. Through numerical simulations, they demonstrate 
that their algorithm is not sensitive to measurement noise, can achieve high computational efficiency, and accurately localize source even without any permissible region constraint.

Khan et al. develop an algorithm to estimate a single-line medial axis, the basis for computation of band profile representative of intensity distribution over chromosomes. They find their algorithm to be robust and capable of estimating a satisfactory single-line medial axis and band profile to be a good representative of intensity levels in different regions of chromosomes.

Gibson et al. present an automated method for nonrigid registration of the optical nerve head surfaces extracted from segmented 3-D OCT images. They illustrate construction of an average optical nerve head shape across an illustrative dataset.

Jo et al. report early favorable results from an animal study designed to measure the capacity of this approach for diagnosing oral cancer in a hamster cheek pouch model of oral cancer using their recently developed noninvasive imaging system incorporating OCT and fluorescence lifetime imaging to acquire both sets of biomarkers.

The remaining set of papers addresses the issues of image analysis for optical, radiological and histopathological image analyses. Although radiological and optical image analyses have attracted much of the attention in the past decades and enormous strides have been made [1], there are several challenging problems still remaining. Histopathogical image analysis, on the other hand, is truly an emerging area growing at an accelerated pace, especially after the introduction of commercially available whole-slide scanners. The size, complexity, scale, and biological richness of these images constitute both the advantages and challenges of this exciting field [2], [3].

Jung et al. present a novel watershed-based method for segmentation of cervical and breast cell images by formulating the segmentation of clustered nuclei as an optimization problem. By using a priori knowledge with respect to the shape of nuclei, they solve the optimization problem.

$\mathrm{Lu}$ et al. propose an automated retinal-layer-segmentation technique for OCT images. Their experiments with four objects demonstrate that their proposed technique segments the image into five layers accurately.

Samsi et al. develop a follicle detection method from immunohistochemically stained slides of follicular lymphoma using texture and color information to mimic the process that a human expert might use to identify follicle regions. They compare system-generated results with expert-generated ground truth and show promising results.

Sertel et al. address computer-aided detection of centroblasts for follicular lymphoma grading using adaptive likelihoodbased cell segmentation. The proposed system uses a unitone conversion to obtain a single-channel image that has the maximum contrast and from the resulting image, a cell-likelihood image is generated in an adaptive way that is robust to variations using the estimated parameters associated with hematoxylin stain concentration of pixels.

Cooper et al. illustrate the challenges associated with analyzing and integrating large-scale image datasets with molecular characterizations. They present their methodology for address- ing these challenges in the context of a study on diffuse glioma brain tumors, and present a motivating example for correlating nuclear morphometry with molecular characterization of glioblastomas.

Tian et al. investigate an approach for automatic liver segmentation from CT scans based on a statistical-shape model integrated with an optimal-surface-detection strategy. They apply their method to MICCAI 2007 liver segmentation challenge datasets.

Sadeghi Naini et al. propose a technique to enhance the quality of intraoperative ultrasound images of a deflated lung undergoing minimally invasive tumor-ablative procedure. The proposed technique employs information of a deflated lung's computed tomography image constructed preoperatively in order to enhance those of the intraoperative ultrasound images.

\section{ACKNOWLEDGMENT}

The guest editors would like to thank Prof. B. Wheeler, Editorin-Chief of TBME for his encouragement, advice, and continuous support from the conceptualization of the special issue idea and throughout the review process. They are also extremely grateful to the reviewers who carefully reviewed the submissions and provided extremely valuable and constructive critiques while providing all the feedback in a timely manner even after unexpected delays in travel due to the volcanic eruption in Iceland and during several proposal submission deadlines.

MetiN N. GURCAN, Guest Editor
The Ohio State University
Columbus, OH 43210 USA
metin.gurcan@ osumc.edu

BADRINATH ROYSAM, Guest Editor Rensselaer Polytechnic Institute Troy, NY 12180 USA roysam@ecse.rpi.edu

ATAM P. DHAWAN, Guest Editor
New Jersey Institute of Technology
Newark, NJ 07102-1982 USA
dhawan@adm.njit.edu

LIHONG V. WANG, Guest Editor Washington University in St. Louis St. Louis, MO 63130 USA hwang@biomed.wustl.edu

\section{REFERENCES}

[1] A. Dhawan, Medical Image Analysis. New York: Wiley-Interscience, 2003.

[2] M. Gurcan, L. Boucheron, A. Can, A. Madabhushi, N. Rajpoot, and B. Yener, "Histopathological image analysis: A review," IEEE Rev. Biomed. Eng., vol. 2, pp. 147-171, 2009.

[3] Y. Al-Kofahi, W. Lassoued, W. Lee, and B. Roysam, "Improved automatic detection \& segmentation of cell nuclei in histopathology images," IEEE Trans. Bio-Med. Eng., vol. 57, no. 4, pp. 841-852, Apr. 2010. 


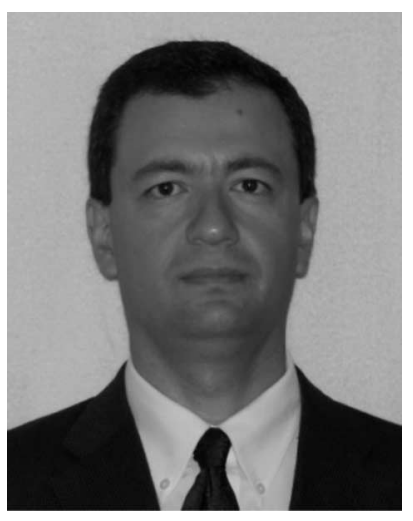

Metin N. Gurcan (M'90-SM'05) received the B.Sc. and Ph.D. degrees in electrical and electronics engineering from Bilkent University, Ankara, Turkey, and the M.Sc. degree in digital systems engineering from the University of Manchester Institute of Science and Technology, Manchester, U.K.

During 1996-1997, he was a Visiting Researcher at the University of Minnesota, Minneapolis. From 1999 to 2001, he was a Postdoctoral Research Fellow and later a Research Investigator in the Department of Radiology at the University of Michigan, Ann Arbor. Prior to joining the Ohio State University, he worked as a Senior Researcher and Product Director at a high-tech company, specializing in computer-aided detection and diagnosis of cancer from radiological images. He is an Associate Professor at the Biomedical Informatics Department, The Ohio State University, Columbus. He currently manages several image analysis related projects funded by the National Cancer Institute, National Library of Medicine, American Cancer Society, Department of Defense and Children's Neuroblastoma Cancer Foundation. He teaches tutorials IEEE International Conference of Image Processing and International Conference on Acoustics, Speech, and Signal Processing, and is the Organizer of and Lecturer at several meetings, workshops, special focus sessions at leading conferences in areas of image analysis.

Dr. Gurcan is a Senior Member of International Society for Optical Engineers and Radiological Society of North America. He is the recipient of the British Foreign and Commonwealth Organization Award, Children's Neuroblastoma Cancer Foundation Young Investigator Award, and National Cancer Institute's caBIG Embodying the Vision Award.

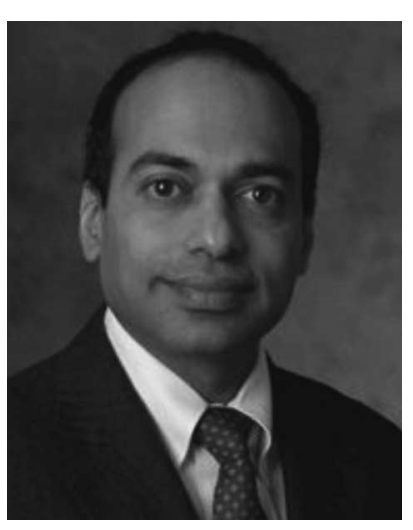

Badrinath Roysam (SM'89) received the B.Tech degree in electronics engineering from the Indian Institute of Technology, Chennai, India, in 1984, and the M.S. and D.Sc. degrees from Washington University, St. Louis, in 1987, and 1989, respectively.

He has been at the Rensselaer Polytechnic Institute, Troy, New York since 1989, where he is currently a Professor in the Electrical, Computer, and Systems Engineering Department. He is an Associate Director of the Center for Subsurface Sensing and Imaging Systems (CenSSIS)—a multiuniversity National Science Foundation-sponsored engineering research center, and Codirector of the Rensselaer Center for Open Source Software. He also holds an appointment in the Biomedical Engineering Department. His current research interests include in the areas of multidimensional biomedical image analysis with, biotechnology automation, optical instrumentation, high-speed and real-time computing architectures, and parallel algorithms.

Dr. Roysam is a Senior Member of the Microscopy Society of America, International Society for Analytical Cytology, Society for Neuroscience, and the Association for Research in Vision and Ophthalmology. He is an Associate Editor for the IEEE TRANSACTIONS ON BIOMEDICAL ENGINEERING and IEEE TRANSACTIONS ON INFORMATION TECHNOLOGY IN BIOMEDICINE. 


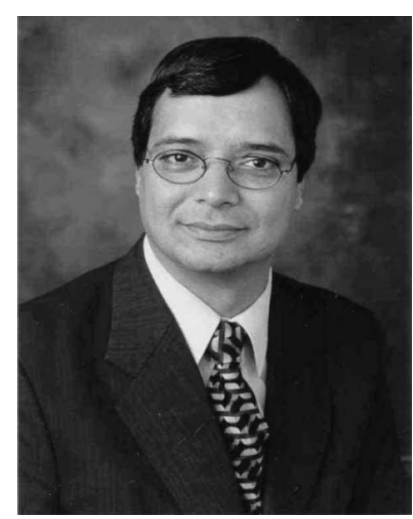

0Atam P. Dhawan (M'79-SM'90-F'04) received the Bachelor's and Master's degrees from the Indian Institute of Technology, Roorkee, India, and the Ph.D. degree from the University of Manitoba, Manitoba, Canada, all in electrical engineering.

From 1985 to 2000, he held faculty positions in Electrical and Computer Engineering, and Radiology Departments at several universities including University of Houston, University of Cincinnati, University of Texas (Arlington), and University of Texas Medical Center (Dallas). Currently, he is a Distinguished Professor of Electrical and Computer Engineering Department with joint appointment in Biomedical Engineering at the New Jersey Institute of Technology, Newark. His research work has been funded by National Institutes of Health (NIH), National Science Foundation, and several industries. He is the author or coauthor of 200 research articles in refereed journals, books, and conference proceedings. His current research interests are medical imaging, multimodality medical image analysis, multigrid image reconstruction, wavelets, genetic algorithms, neural networks, adaptive learning and pattern recognition.

Dr. Dhawan is the recipient of Commonwealth Fellowship during 1983-1985, Martin Epstein Award in 1984, NIH FIRST Award in 1988, Sigma-Xi Young Investigator Award in 1992, University of Cincinnati Faculty Achievement Award in 1994, the IEEE Engineering in Medicine and Biology Early Career Achievement Award in 1995, and University of Toledo Dorman Distinguished Lecture Award in 1999. He is Senior Editor of the IEEE TRANSACTIONS OF BIOMEDICAL ENGINEERING (TBME) and Editor-In-Charge of IEEE TBME Letters. He was the Chair of the "Emerging Technologies Committee" of the IEEE-Engineering in Medicine and Biology Society from 1997 to 1999, and from 2009 to 2010. He was the Chair of the "New Frontiers in Biomedical Engineering" Symposium at the World Congress 2000 on Medical Physics and Biomedical Engineering. He was the Conference Chair of the IEEE 28th International Conference of Engineering in Medicine and Biology Society held from August 30 to September 3, 2006 in New York. He was an American Liaison and EMBS Representative in the Steering Committee of International Symposium of Biomedical Imaging during 2009-2011. He has chaired numerous NIH review panels including the NIH Biomedical Computing and Health Informatics Chartered Study Section.

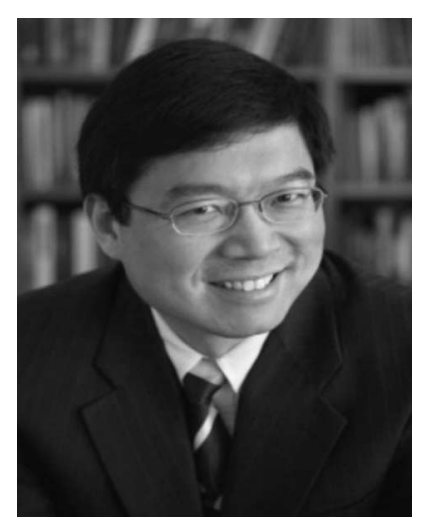

Lihong V. Wang (M'96-SM'00-F'06) received the Ph.D. degree from Rice University, Houston, Texas under the tutelage of Drs. Robert Curl, Richard Smalley, and Frank Tittel.

He is the holder of the Gene K. Beare Distinguished Professorship of Biomedical Engineering at Washington University in St. Louis, St. Louis, MO, and directs the Optical Imaging Laboratory and the Imaging Division. His Monte Carlo model of photon transport in scattering media has been used worldwide. He is the author of one of the first textbooks in the field of biomedical optics. $\mathrm{He}$ is the editor of the first comprehensive book on photoacoustic tomography. He is the author or coauthor of more than 220 peer-reviewed journal articles and delivered more than 250 invited talks.

Dr. Wang is a Fellow of the AIMBE, OSA, and SPIE. He is the Editor-in-Chief of the Journal of Biomedical Optics. He chairs the annual conference on Photons plus Ultrasound and chaired the 2010 Gordon Conference on Lasers in Medicine and Biology and the 2010 OSA Topical Meeting on Biomedical Optics. He also chairs the International Biomedical Optics Society. He is a Chartered Member on National Institutes of Health (NIH) study section. He is the Founding Chair of the Scientific Advisory Boards for two companies commercializing photoacoustic tomography. He is the recipient of NIH FIRST Award, National Science Foundation CAREER Award, and the 2010 Joseph W. Goodman Book Writing Award. His laboratory invented or discovered frequency-swept ultrasound-modulated optical tomography, dark-field confocal photoacoustic microscopy (PAM), optical-resolution PAM, photoacoustic Doppler sensing, photoacoustic reporter gene imaging, focused scanning microwave-induced thermoacoustic tomography, exact reconstruction algorithms for photoacoustic or thermoacoustic tomography, sonoluminescence tomography, Mueller-matrix optical coherence tomography, optical coherence computed tomography, and oblique-incidence reflectometry. 